This is an Accepted Manuscript of an article published by Taylor \& Francis in Scandinavian Journal of Urology, 2020, 54(3), 201-207. Available online: https://doi.org/10.1080/21681805.2020.1750474

\title{
Urine Colour as an Indicator for Anastomotic Leakage after Robot-Assisted Radical Prostatectomy
}

Running title: Urine colour and anastomotic leakage

Jarno Riikonen ${ }^{1,2}$, Tomi Pakarainen ${ }^{1,2}$, Aino Siltari ${ }^{1}$, Juha-Pekka Pienimäki ${ }^{1,3}$, Juha Koskimäki ${ }^{1,2}$, Teemu J Murtola ${ }^{1,2,4^{*}}$

1. Tampere University, Faculty of Medicine and Health Technology, Tampere, Finland

2. Department of Urology, Tampere University Hospital, Tampere, Finland

3. Medical Imaging Center, Tampere University Hospital, Tampere, Finland

4. Department of Surgery, Seinäjoki Central Hospital, Seinäjoki, Finland.

*Corresponding author: Teemu J Murtola, Department of Urology, Tampere University Hospital, PL 2000, 33521 Tampere, Finland, teemu.murtola@tuni.fi

Word count abstract: 250

Word count text (without abstract, references and legends): 2923 


\section{Abstract}

Objectives: To determine whether macroscopic hematuria predicts urethrovesical anastomotic leakage after robot-assisted laparoscopic radical prostatectomy (RALP) as well as a cystogram.

Patients and methods: Participants were recruited before cystogram and catheter removal 5 to 14 days after RALP surgery. Urine colour in the collection bag was classified according to a three-step scale (clear, light red and dark red) and leakages in cystogram were graded with a four-step scale (Grade 0-3). Diagnostic accuracy parameters were calculated for urine colour. A multivariate logistic regression model was used to evaluate other leakage risk factors.

Results: Of 214 patients, 201 (94\%) had clear, 6 (3\%) had light red and 7 (3\%) had dark red coloured urine. In the cystogram, 20 (9\%) patients had leakage; 14 had Grade 1, 5 Grade 2 and 1 Grade 3 leakage. Overall, specificity and sensitivity of urine colour in predicting anastomotic leakage were 0.97 (95\% CI 0.95-100) and 0.38 (95\% CI 0.17-0.59), respectively. Negative and positive predictive values were $94 \%$ and $62 \%$, respectively.

Other significant risk factors for anastomotic leakage were previous transurethral resection or radiation therapy to the prostate, non-waterproof anastomosis at surgery, postoperative pelvic hematoma, long catheterization and surgeon's inexperience. In patients with no other risk factors, test sensitivity improved to 0.80 (95\% CI $0.45-1.15)$ and negative and positive predictive values to $99 \%$ and $44 \%$, respectively.

Conclusion: Our prospective single-arm trial indicates that in patients with clear urine and no other risk factors for anastomotic leakage, a cystogram examination before urethral catheter removal can be safely omitted.

$\underline{\text { Key words: }}$ robot-assisted radical prostatectomy, cystogram, urine colour, anastomosis, anastomotic leakage 


\section{Introduction}

Cystogram imaging is a common method to rule out urethrovesical anastomotic leakage after radical prostatectomy. Since the incidence of anastomotic leakage is low, many surgeons do not recommend the taking of a cystogram before urinary catheter removal [1,2]. As a way of reducing unnecessary imaging, it has been postulated that a cystogram should be performed only in patients at risk for urethrovesical anastomotic leak [3]. On the other hand, the cystogram is still widely used, because catheter removal during anastomotic leakage exposes the individual to complications such as uroperitoneum, peritonitis, ileus and renal function deterioration [4,5].

Risk factors for urethrovesical anastomosis leakage can be classified as pre-, intra- and post-operative factors. According to previous trials, the preoperative risk factors include obesity, ischaemic heart disease, previous transurethral resection of prostate (TURP), large prostate, lower urinary tract symptoms (LUTS) and previous pelvic radiation [6-9]. The intraoperative risk factors include excessive bleeding, mucosal eversion, wide bladder neck opening, need for bladder neck reconstruction, technically difficult anastomosis, tension in anastomosis and an unsatisfactory flush test result perioperatively [3,7,9-12]. In addition, an anastomotic leak is more common during the early phase of the learning curve of the procedure, decreasing as the surgeon gains experience with the procedure [13-14]. The postoperative factors which increase the anastomotic leak are postoperative urinary tract infection [15] and pelvic para-anastomotic haematoma [15]. In addition, a bloody urine colour is a predictive factor for urethrovesical anastomotic leakage [16-17].

Cystogram imaging is the golden standard for the determination of anastomotic leakage. Nevertheless, it is not known whether failing to perform a cystogram would be both feasible and safe based on the leakage risk factor estimation. In this study, we determined whether macroscopic haematuria of any grade predicts urethrovesical anastomotic leakage after robot-assisted laparoscopic radical prostatectomy (RALP). 


\section{Patients and Methods}

\section{Patients and Collected Information}

We prospectively recruited 227 men scheduled for urinary catheter removal after RALP performed in the Tampere University Hospital, Tampere, Finland between June 2015 and January 2017. The Ethics Committee of the Pirkanmaa Hospital District, Tampere, Finland approved the study protocol (identification number: R15042). Written consent was obtained from all participants and study was performed according to the Declaration of Helsinki. The study protocol has been registered at ClinicalTrials.gov (NCT02526589). Inclusion criteria of the study were RALP operation 5 to 14 days before catheter removal and signing of informed consent form.

We recorded previously reported possible risk factors for anastomotic leakage, e.g. size of the removed prostate, surgical data, hospitalization length and complications until urethral catheter removal. Surgical data included surgeon's experience, operation length, possible lymphadenectomy, nerve-sparing, use of haemostatic agents, intraoperative blood loss and haematocrit value before surgery and on the 1st postoperative day. Data was also completed with information on patient demographics, comorbidities, medication use and previous operations in the pelvic area.

\section{RALP Technique}

RALP procedures were carried out with the aid of a four-arm da Vinci S robot (da Vinci; Intuitive Surgical, Sunnyvale, CA, USA). The detailed surgical technique has been described previously [17]. A double-layer posterior reconstruction of rhabdosphincter was performed using 3-0 barbed V-lock suture. Urethrovesical anastomosis was reconstructed using the Van Velthoven technique with 3-0 poliglecaprone threads on a UR6 needle (Monocryl; Ethicon, Somerville, NJ, USA). A two-way silicone Ch18 Foley urethral catheter (Folysil; Coloplast, Denmark) was used. An urethral catheter was fenestrated at the site of urethrovesical anastomosis to decrease the risk of urethrovesical anastomosis leak [17]. Intraoperative flush test of the anastomosis was performed by filling the 
bladder with $100 \mathrm{ml}$ of saline. If the anastomosis was not watertight, the surgeon either re-performed the anastomosis, placed patch sutures or did nothing, depending on the surgeon's clinical decision. No drains were routinely placed. The patients used low-molecular-weight heparin for thromboprophylaxis for ten days after surgery as recommended by hospital guidelines at the time.

\section{Estimation of Urine Colour}

The patients were recruited to the study when they arrived from home to the urological outpatient clinic for a routine cystogram examination and urethral catheter removal within 5 to 14 days after surgery. After the patient signed the informed consent, a nurse visually estimated the urine colour according to a 3-step scale: clear, light red or dark red. The nurse also took a photograph of the urinary collection bag for later validation (Figure 1). Subsequently, two researchers (JR and TP) re-analysed the photographs independently and blinded to cystogram result.

\section{Cystogram}

The cystogram was performed by filling the bladder with $200 \mathrm{ml}$ of iodinated contrast medium (Omnipaque $140 \mathrm{ml} \mathrm{I/ml:} \mathrm{Amersham} \mathrm{Health,} \mathrm{Princeton,} \mathrm{NJ,} \mathrm{USA).} \mathrm{Anteroposterior,} \mathrm{lateral} \mathrm{and}$ oblique views were taken to detect possible extravasation. A clinical radiologist examined the images blinded to the urinary colour. Cystogram images were downloaded to the hospital's database and analysed afterward by a blinded study radiologist (JPP) who classified extravasations as negative for leakage (Grade 0), extraperitoneal leakage extending less than $6 \mathrm{~cm}$ (Grade 1), extraperitoneal leakage extending more than $6 \mathrm{~cm}$ (Grade 2) and intraperitoneal leakage (Grade 3) (Figure 2) [19]. This classification was used in the analysis.

Urologists in the Tampere University Hospital outpatient clinic checked the cystogram images and radiologist's reports. If there was no or only minor extravasation at the urethrovesical anastomosis, the catheter was removed. If there was a marked extravasation (Grade 2-3), the catheter was left for 1-2 weeks according to the urologist's decision. 


\section{Power Calculation}

We assumed that there would be a vesicourethral anastomosis leakage rate of $8 \%$ based on a previously published study [17]. In order to reach a of 0.80 sensitivity and specificity assuming this leakage rate, we estimated that we would need 201 patients or 17 anastomotic leakages. To allow for dropouts, we set the enrolment target at 222 patients.

\section{Statistical Analysis}

The main analysis estimated predictive accuracy of macroscopic hematuria of any grade. Planned subgroup analyses were performed by grade of hematuria and grade of leakage. We used an Excel-based diagnostic calculator [20] to estimate test sensitivity, specificity, positive and negative predictive values as well as positive and negative likelihood ratios for urine colour as predictor of urethrovesical anastomotic leakage in the routine cystogram after RALP. Sensitivity i.e. true positive value indicates the proportion of detected positive cases from confirmed positive cases. Specificity i.e. true negative rate indicates the proportion of detected negative tests from actually negative cases. Positive predictive value refers to the percentage of confirmed positive cases from cases in which urine colour test was positive and the negative predictive value refers to the percentage of confirmed negative cases from cases in which the urine colour test was negative. Likelihood ratios were used to calculate the probability of leakage if the colour test result was positive or negative. The prevalence for leakage in the study population was used as the leakage risk in the probability calculation. Mann-Whitney U test was used to calculate the statistical difference for continuous variables and Pearson Chi-Square test for categorical variables.

A multivariate logistic regression model was used to estimate the significance of other putative risk factors for predicting urethrovesical anastomotic leakage. First, all possible risk factors for anastomotic leakage were tested in multivariate analysis (model 1). All statistically significant risk factors in model 1 were added to the final multivariate model. Variables included in the final model 
were urine colour, age, surgeon's experience (less or more than 100 operations), prior TURP, intraoperative anastomotic leak and technically difficult anastomosis.

P-value less than 0.05 was considered statistically significant. All analyses were conducted using IBM SPSS software (version 24).

\section{Results}

\section{Population Characteristics}

A total of 227 patients were recruited. Thirteen patients were excluded before the analysis; 11 due to a leakage in a previous cystogram and the present cystogram which was performed more than 14 days after surgery, one having undergone bladder diverticulectomy (not RALP) and one because of medium contrast allergy. Therefore, 214 patients were included in the final analysis.

A total of 201 (94\%) patients had clear urine, six (3\%) patients had light red and 7 (3\%) patients had dark red urine colour (Table 1). In the cystogram, 20 (9\%) patients had leakage. The leakage grades were distributed to Grade 1, Grade 2 and Grade 3 in 14 (7\%), 5 (2\%) and 1 (0.5\%) patients, respectively (Table 1$)$.

There was no difference in mean PSA levels, prostate volume, smoking or heavy alcohol consumption, BMI, comorbidities (hypertension, diabetes or ischaemic heart disease) or additional medication use between men with or without leakage (Table 2). Men with leakage had a greater haematocrit decline, more bleeding complications and bloody urine, longer catheterization, more intraoperative anastomosis leak and the surgery had been performed by a less experienced surgeon as compared to men without post-operative leakage (Table 2). Patients with leakage also had had more often prior TURP and pelvic radiation. 


\section{Distribution Between Urine Colour and Anastomotic Leakage}

A total of 5 men (2.6\% of true negatives) were false positive, i.e. they had bloody urine without leakage in cystogram, whereas 12 men (60\% of true positives) were false negative, i.e. having clear urine despite leakage in their cystogram (Table 1).

Overall specificity and sensitivity of bloody urine colour in predicting anastomotic leakage were 0.97 (95\% CI 0.95-100) and 0.38 (95\% CI 0.17-0.59), respectively. Positive and negative predictive values were $62 \%$ and $94 \%$, respectively. The probability to have leakage if the test result was positive was $60 \%$. If the test was negative, then the probability was $6 \%$. A dark urine colour was observed only in men with leakage (Table 1). Dark red urine colour $(n=7)$ alone displayed similar sensitivity and specificity levels (0.35 and 1.00, respectively) as urine colour in general, but the positive predictive value was improved to $100 \%$. Test sensitivity was slightly better in predicting Grade 2-3 leakage (0.50) while specificity remained high (0.97). The positive predictive value was $38 \%$ while the negative predictive value was $98 \%$.

In addition to the presence of bloody urine, other significant risk factors for anastomotic leakage were previous TURP or radiotherapy to the pelvis, anastomosis not being watertight at surgery or problems (re-suturing) when creating the anastomosis, postoperative hematoma in the pelvis, bleeding complications, any Clavien 3 complications and surgeon's inexperience (Table 2). In addition, men with leakage were older than men without leakage.

Limiting the analysis to cases without the above-mentioned risk factors $(n=199)$ for anastomotic leakage markedly improved test sensitivity; specificity and sensitivity of the urine colour test in this subgroup were 0.97 (95\% CI 0.95-1) and 0.8 (95\% CI 0.45-1.15), respectively. Positive and negative predictive values were $44 \%$ and $99 \%$, respectively. The probabilities for leakage after positive and negative test results were $62 \%$ and $1 \%$, respectively. 


\section{Logistic Regression Model}

When the analysis was conducted to determine the impact of urine colour and a summary variable including other significant leakage risk factors for risk of post-operative leakage, both were statistically significant ( $<<0.001$ in both). In the multi variate analysis including other risk factors, urine colour $(\mathrm{p}<0.001)$, surgeon's experience $(\mathrm{p}=0.009)$, prior TURP $(\mathrm{p}=0.02)$ and leak at intraoperative flush test $(\mathrm{p}=0,002)$ all had an independent, statistically significant risk association with leakage and these results were not modified by grade of urine colour (light and dark red).

\section{Discussion}

Our study shows that bloody urine colour predicts urethrovesical anastomosis leakage with high specificity but low sensitivity after RALP although taking into account other risk factors for leakage does improve the sensitivity. Therefore, if the patient voids clear urine then it is feasible to omit cystography, especially if no leakage risk factors are present.

Specificity i.e. the true negative rate was 0.97 or more and the negative predictive value was $94 \%$ or more in every analysis, thus based simply on the presence of a clear urine colour, patients without anastomotic leakage could be reliably identified. The probability for leakage after a negative test result was very low, $6 \%$ or less. Thus, we recommend that in majority of the patients voiding clear urine, there is no need for a routine postoperative cystogram; a cystogram should be performed only when urine is coloured or other risk factors exist. A multivariate analysis also supports this recommendation.

The risk factors for leakage were observed to be bloody urine colour, a previous TURP, previous pelvic irradiation, intraoperative anastomosis leak, difficult anastomosis, pelvic haematoma and surgeon's inexperience. According to the multivariate analysis, clinically meaningful risk factors were bloody urine, the experience of the surgeon, prior TURP and leak in intraoperative flush test. In our study patients without any of these risk factors, the urine colour test missed only one leakage; 
negative predictive value stayed in $99 \%$ and probability to have leakage after a negative test result was reduced to $1 \%$.

Macroscopic haematuria at the time of catheter removal has been shown to be a significant predictive factor for anastomotic leak also by other groups [16-17]. However, in previous studies, the surgical technique in use was open retropubic radical prostatectomy, where the anastomosis was sewn with single sutures and the colour of urine was estimated when the patients were still in bed in the hospital. Instead, in our study, all prostatectomies are done using a robot-assisted technique, continuous sutures are used for the anastomosis and patients are mobilised and discharged early, usually on the first postoperative day. They arrive from home for urethral catheter removal and thus results from the previous studies may not be generalised per se to the more modern clinical practice.

Our study shows that macroscopic hematuria predicts urethrovesical anastomotic leakage after RALP with reasonable accuracy. However, it still misses small proportion of leakages, especially in men with risk factors for leakage. The most adverse complication, which might follow anastomotic leakage, is uroperitoneum causing peritonitis [21]. Some evidence also supports the hypothesis that anastomotic leakage might induce anastomotic strictures [22,23]. Furthermore, it seems that incidence of incontinence is increased after anastomotic leakage [24]. Relying solely on urine colour risks missing some leakages, which may increase occurrence of these harms. On the other hand, it removes the need for cystogram imaging unnecessary for most, thus opening potential for reducing radiation exposure and costs.

We estimated the prevalence of anastomotic leakage as 9\%. In recently published studies, the incidence of anastomotic leakage after RALP has varied from $2.9 \%$ [21] to $3.9 \%$ [3] up to $13.6 \%$ [18], i.e. a similar incidence as found here. However, even anastomotic leakage rates as high as $26 \%$ have been reported recently [6]. A cystogram was performed in all patients in our study, thus also 
small leakages (Grade 1) were detected. An evaluation of the clinical significance of such small leakages is beyond the scope of this study.

Surgeon's experience also affects the level of anastomotic leakage rate. Surgeon's experience inversely associates with the rate of anastomotic leakage, which can be seen from the learning curve of Dr Vipul Patel: during his first 300 RALPs, the leakage rate was 4\%, in procedures from 300 to 600 the leakage rate was $2.6 \%$ and from 2100 to 2400 leakage rate was a mere $0.3 \%$ [13]. This phenomenon can be seen also in our study: in $65 \%$ of the cases when leakage occurred, the surgeon was inexperienced. Overall, inexperienced surgeons performed $20 \%$ of the RALPs in our study.

The strength of our study is that it is a prospective and registered study. We also blinded all operators; the nurses and the urologists who estimated urine colour and the radiologists who analysed cystogram images.

There were also some limitations. The size of our study is relatively small and RALPs were performed in a single hospital. However, we were able to achieve the number of patients and leakages demanded by our power calculation. We did not have a strict protocol for management of anastomotic leakage. Our recommendation was to remove the urethral catheter if $2 \mathrm{~cm}$ or smaller contrast fluid extravasation was found in the cystogram. In fact, the urologists were allowed to make an independent clinical decision. However, we wished to test the hypothesis that urine colour would be a marker for leakage and therefore could be used to decide whether a cystogram would be needed or not. The aim was not to investigate the clinical significance of any leakages detected, thus, strict protocol how to practice in case of leakage was omitted.

Urine colour was visualised only by visual inspection. We tried to develop some kind of computerbased colour analysis method but this was not successful. However, visual colour analysis only by the human eye may also be a strength because it makes the clinical evaluation more straightforward. Furthermore, intra-observer variability was controlled by taking a photograph from the urine 
collection bags after the nurses' visual evaluations and two urologists blinded and independently evaluated the colour from these photographs.

\section{Conclusions}

Our prospective trial showed that a bloody urine colour at 5 to 14 days after RALP is a predictor for urethrovesical anastomosis leakage. Conversely, if urine is clear and there are no other risk factors for anastomotic leakage, then performing a cystogram prior to urethral catheter removal is not necessary.

\section{Acknowledgements}

We would like to acknowledge nurses Jaana Saarinen and Jari Peltonen, who took photographs and analysed the urinary colours, Mika Helminen, who helped us in the statistical analysis, and Dr Ewen MacDonald, for improvements to language of the manuscript. This study was funded by Expert Responsibility Area of the Pirkanmaa Hospital District (grant number 9X032) and Cancer Society of Finland (grant number 25024194).

\section{Disclosure of interest}

Tomi Pakarainen, Aino Siltari, Juha-Pekka Pienimäki and Juha Koskimäki: Nothing to declare.

Jarno Riikonen: Reimbursements for travel and conferences: Swanmedica. Trial participation: Bayer, Orion.

Teemu Murtola: Consultation fees from Astellas and Janssen. Lecture fees from Astellas and Janssen. Reimbursements for travel and conferences: Janssen

\section{Authors' contribution}

Jarno Riikonen, Tomi Pakarainen, Juha-Pekka Pienimäki, Juha Koskimäki and Teemu Murtola performed the research. Jarno Riikonen, Tomi Pakarainen and Teemu Murtola designed the research 
study. Jarno Riikonen, Tomi Pakarainen, Aino Siltari, Juha-Pekka Pienimäki and Teemu Murtola analysed the data. Jarno Riikonen, Aino Siltari and Teemu Murtola wrote the manuscript. All authors have approved the final version of the manuscript.

\section{References}

1. Gratzke C, Dovey Z, Novara G, et al. Early Catheter Removal after Robot-assisted Radical Prostatectomy: Surgical Technique and Outcomes for the Aalst Technique (ECaRemA Study). Eur Urol. 2016;69(5):917-923.

2. Guru KA, Seereiter PJ, Sfakianos JP, et al. Is a cystogram necessary after robot-assisted radical prostatectomy? Urol Oncol. 2007;25(6):465-467.

3. Yadav R, Bansal S, Gupta NP. Selective indication for chec cystogram before catheter removal following robot assisted radical prostatectomy. Indian J Urol. 2016;32(2):120-123.

4. Mochtar CA, Kauer PC, Laguna MP, et al. Urinary leakage after laparoscopic radical prostatectomy: a systematic review. J Endourol. 2007;21(11):1371-1379.

5. Shak G, Vogel F, Moinzadeh A. Nephroureteral stent on suction for urethrovesical anastomotic leak after robot-assisted laparoscopic prostatectomy. Urology. 2009;73(6):13751376.

6. Tillier C, van Muilekom HAM, Bloos-van der Hulst J,et al.Vesico-urethral anastomosis (VUA) evaluation of short- and long-term outcome after robot-assisted laparoscopic radical prostatectomy (RARP): selective cystogram to improve outcome. J Robot Surg. 2017;11(4):441-446.

7. Tyritzis SI, Katafigiotis I, Constantinides CA. All you need to know about urethrovesical anastomotic urinary leakage following radical prostatectomy. J Urol. 2012(2);188:369-376. 
8. Eandi JA, Link BA, Nelson RA et al. Robotic assisted laparoscopic salvage prostatectomy for radiation resistant prostate cancer. J Urol. 2010;183(1):133-137.

9. Ramsden AR, Chodak GW. Can leakage at the vesico-urethral anastomosis be predicted after radical retropubic prostatectomy? BJU Int. 2004;93(4):503-506.

10. Fenig DM, Slova D, Lepor H. Postoperative blood loss predicts the development of urinary extravasation on cystogram following radical retropubic prostatectomy. J Urol. 2006;175(1):146-150.

11. Gnanapragasam VJ, Baker P, Naisby GP, et al. Identification and validation of risk factors for vesicourethral leaks following radical retropubic prostatectomy. Int J Urol. 2005;12(11):948952.

12. Ischia JJ, Lindsay S. Is a cystogram necessary after radical prostatectomy? ANZ J Surg. 2005;75(9):825-827.

13. Coelho RF, Palmer KJ, Rocco B, et al. Early complication rates in a single-surgeon series of 2500 robotic-assisted radical prostatectomies: report applying a standardized grading system. Eur Urol. 2010;57(6):945-952.

14. Rassweiler J, Seemann O, Hatzinger M, et al. Technical evolution of laparoscopic radical prostatectomy after 450 cases. J Endourol. 2003;17(3):143-154.

15. O'Malley RL, Telegrafi S, Laze J, et al. Para-anastomotic haematoma volume predicts the presence of anastomotic extravasation after radical retropubic prostatectomy. BJU Int. 2010;105(1):34-36.

16. Schenck M, Schneider T. New standardization of checking the vesicourethral anastomosis for tightness following radical prostatectomy with dynamic transrectal ultrasound: can this new technique replace the traditional postoperative cystogram? World J Urol. 2011;29(5):651-655. 
17. Schoeppler GM, Zaak D, Schuhmann P, et al. Urine colour after radical prostatectomy predicts urinary leakage at the vesicourethral anastomosis. Scand J Urol Nephrol. 2012;46(5):337-342.

18. Riikonen J, Kaipia A, Matikainen M, et al. Side-fenestrated catheter decreases leakage at the urethrovesical anastomosis after robot-assisted laparoscopic radical prostatectomy. Scand J Urol. 2014;48(1):21-26.

19. Williams TR, Longoria OJ, Asselmeier S, et al. Incidence and imaging appearance of urethrovesical anastomotic urinary leaks following da Vinci robotic prostatectomy. Abdom Imaging. 2008;33(3):367-370.

20. MacEneaney PM, Malone DE. The meaning of diagnostic test results: a spreadsheet for swift data analysis. Clin Radiol. 2000;55(3):227-235.

21. Jacobsen A, Berg KD, Iversen P, et al. Anastomotic complications after robot-assisted laparoscopic and open radical prostatectomy. Scand J Urol. 2016;50(4):274-279.

22. Tyritzis SI, Katafigiotis I, Constantinides CA. All you need to know about urethrovesical anastomotic urinary leakage following radical prostatectomy. J Urol. 2012;188(2):369-376.

23. Surya BV, Provet J, Johanson KE, et al. Anastomotic strictures following radical prostatectomy: risk factors and management. J Urol. 1990;143(4):755-758.

24. Rebuck DA, Haywood S, McDermott K, et al. What is the long-term relevance of clinically detected postoperative anastomotic urine leakage after robotic-assisted laparoscopic prostatectomy? BJU Int. 2011;108(5):733-778.

25. Patil N, Krane L, Javed K, et al. Evaluating and grading cystographic leakage: correlation with clinical outcomes in patients undergoing robotic prostatectomy. BJU Int. 2009;103(8):1108-1110. 


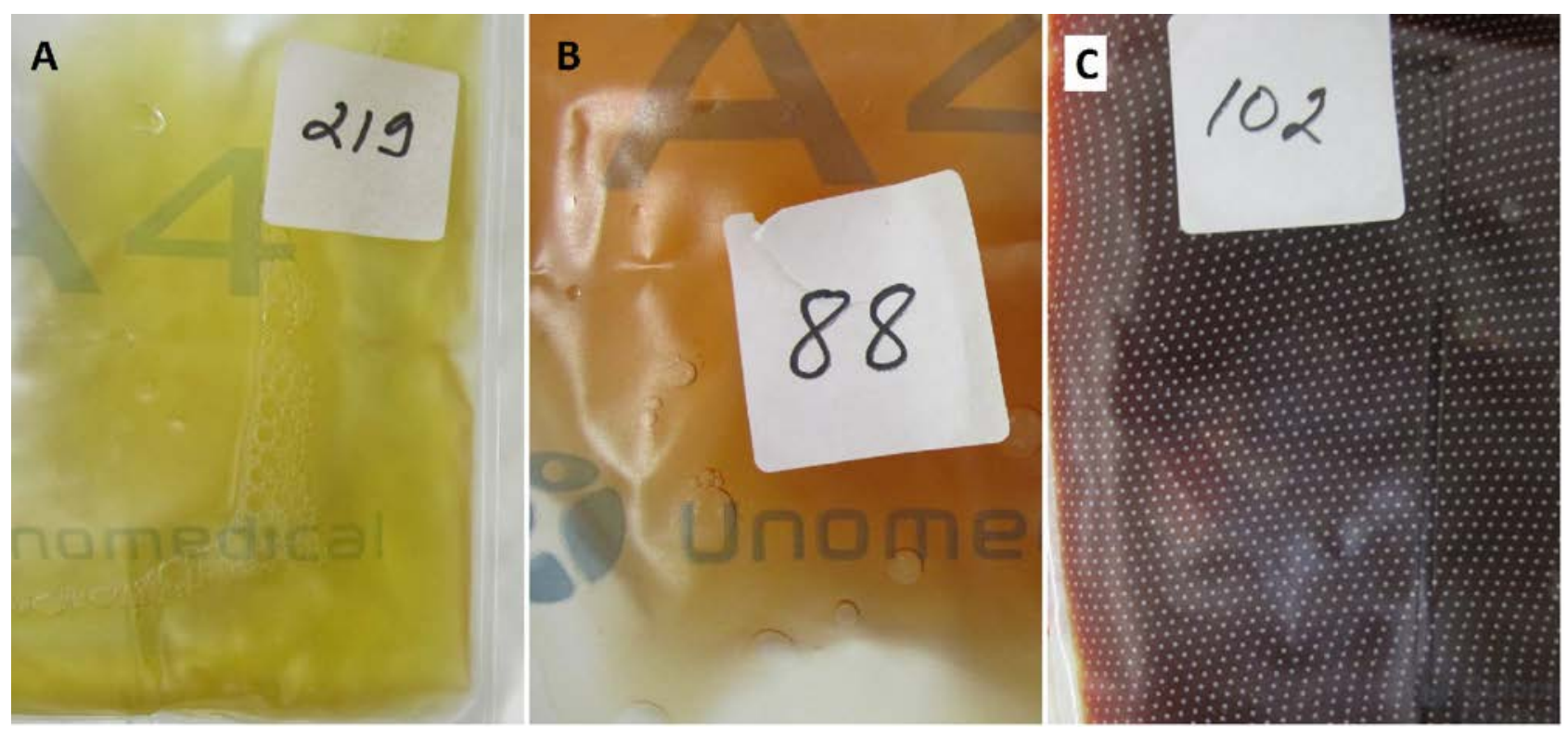

Figure 1. Photographs from urine collection bags from 5 to 14 days after robotic radical prostatectomy. The colours of urine are classified as clear (A), light red (B) and dark red (C). 


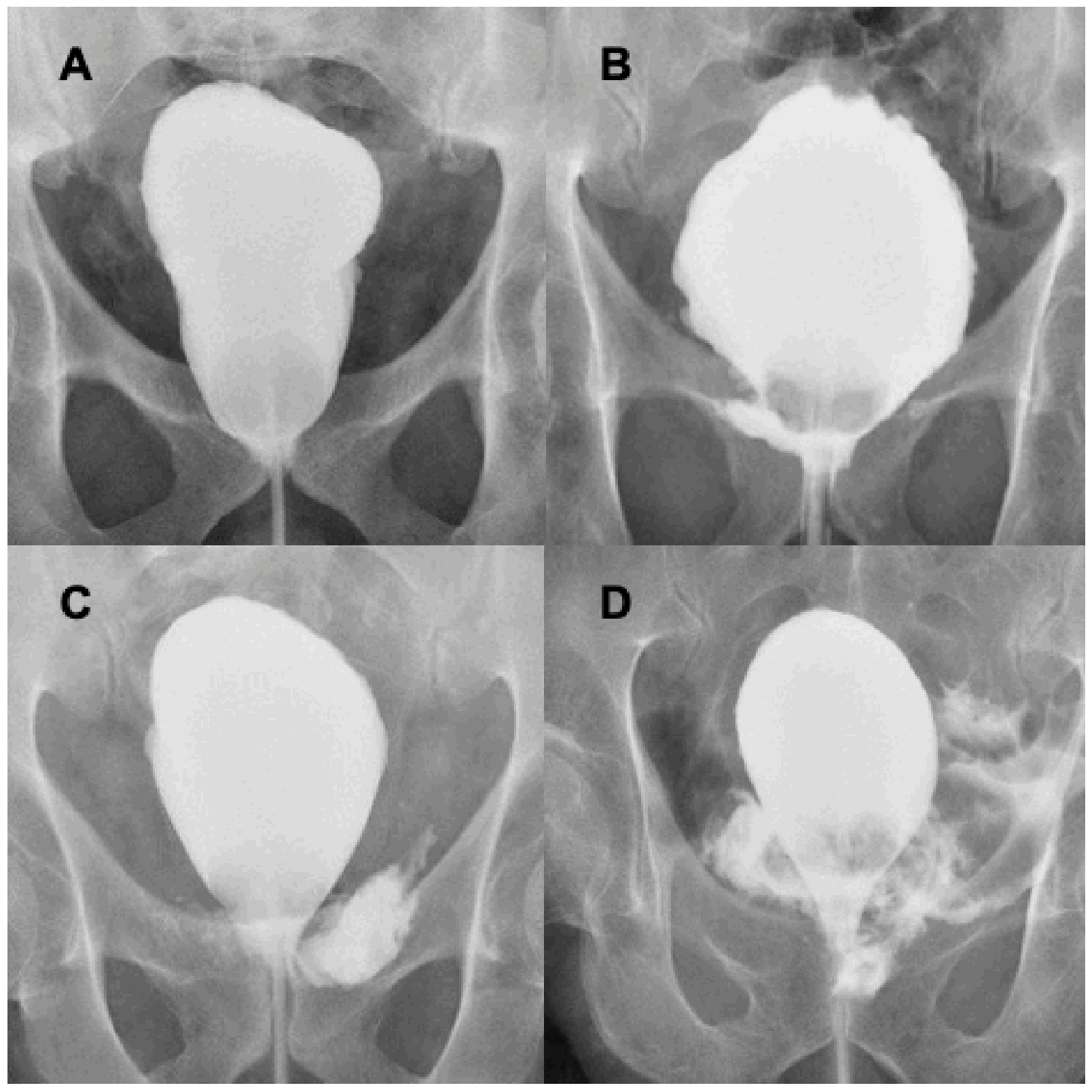

Figure 2. Cystogram images from 5 to 14 days after robotic radical prostatectomy demonstrating no leakage (A), a mild Grade 1 leak to the surgical bed (B), a moderate Grade 2 extraperitoneal leak (C) and a Grade 3 intraperitoneal leakage (D) at urethrovesical anastomosis. 
Table 1. Distribution between the colour of urine and anastomotic leakage from 5 to 14 days postoperatively. ${ }^{*} p$-value for all patients between urine colour and leakages, \#p-value for light red and dark red urine colour and leakeges

\begin{tabular}{|c|c|c|c|c|c|c|c|}
\hline & No leakage & Leakage & Grade 1 & Grade 2 & Grade 3 & $\begin{array}{c}\text { Total } \\
\text { number }\end{array}$ & $P$ \\
\hline $\begin{array}{l}\text { Clear } \\
\text { urine }\end{array}$ & 189 & 12 & 9 & 2 & 1 & 201 & \multirow{2}{*}{$<0.001^{*}$} \\
\hline $\begin{array}{l}\text { Bloody } \\
\text { urine }\end{array}$ & 5 & 8 & 5 & 3 & 0 & 13 & \\
\hline Light red & 5 & 1 & 1 & 0 & 0 & 6 & \multirow{2}{*}{$0.002 \#$} \\
\hline Dark red & 0 & 7 & 4 & 3 & 0 & 7 & \\
\hline $\begin{array}{l}\text { Total } \\
\text { number }\end{array}$ & 194 & 20 & 14 & 5 & 1 & 214 & \\
\hline
\end{tabular}


Table 2. Clinical characteristics, and comparison of all patients (ALL), patients with noleakage (NO-LEAKAGE) and patients with leakage (LEAKAGE) at 5 to 14 days postoperative cystogram. Result are expressed as N (\%). *surgeon was defined as inexperienced if he/she had had less than 100 operation.

\begin{tabular}{|c|c|c|c|}
\hline & ALL & NO-LEAKAGE & LEAKAGE \\
\hline & $\mathrm{N}=214$ & $\mathrm{~N}=194(90.7 \%)$ & $\mathrm{N}=20(9.3 \%)$ \\
\hline Age, years, mean (SD) & $63.8(6.1)$ & $63.5(6.1)$ & $66.3(5.4)$ \\
\hline PSA, mean (SD) & $9.0(6.2)$ & $9.1(6.2)$ & $8.0(6.0)$ \\
\hline Prostate volume, mean (SD) & $39.1(16.8)$ & $38.7(16.7)$ & $42.5(17.3)$ \\
\hline cT3 or higher & $9(4.2)$ & 8 & $1(5.0)$ \\
\hline \multicolumn{4}{|l|}{ Biopsy ISUP grade group } \\
\hline 1 & $81(38)$ & 75 (39) & $6(30)$ \\
\hline 2 & $83(39)$ & $72(37)$ & $11(55)$ \\
\hline 3 & $14(7)$ & $14(7)$ & $0(0)$ \\
\hline 4 & 19 (9) & $16(8)$ & $3(15)$ \\
\hline 5 & $17(8)$ & $17(9)$ & $0(0)$ \\
\hline BMI, mean (SD) & $27.2(3.9)$ & $27.3(3.9)$ & $26.8(4.1)$ \\
\hline Smoking & $23(10.7)$ & $21(10.8)$ & $2(10)$ \\
\hline Heavy alcohol consumption & $18(8.4)$ & $17(8.8)$ & $1(5)$ \\
\hline Prior abdominal surgery & $49(22.9)$ & $41(21.1)$ & $8(40)$ \\
\hline Prior TURP & $7(3.2)$ & $4(2.1)$ & $3(15)$ \\
\hline Prior pelvic radiation & $1(0.5)$ & $0(0)$ & $1(5)$ \\
\hline LUTS & $35(16.4)$ & $30(15.5)$ & $5(25)$ \\
\hline Median lobe & $15(7.0)$ & $12(6.2)$ & $3(15)$ \\
\hline hypertension & $88(41.1)$ & $79(40.7)$ & $9(45)$ \\
\hline diabetes & $30(14.2)$ & $25(12.9)$ & $5(25)$ \\
\hline Ischaemic heart disease & $13(6.1)$ & $11(5.7)$ & $2(10)$ \\
\hline Statin medication & $59(27.6)$ & $50(25.8)$ & $9(45)$ \\
\hline 5-ARI medication & $15(7.0)$ & $14(7.2)$ & $1(5)$ \\
\hline Diabetes medication & $28(13.1)$ & $23(11.9)$ & $5(25)$ \\
\hline Anticoagulant medication & $35(16.4)$ & $28(14.4)$ & $6(30)$ \\
\hline Inexperienced surgeon* & $44(20.6)$ & $31(16.0)$ & $13(65.0)$ \\
\hline Nerve-sparing & $169(79.0)$ & $156(80.4)$ & $13(65)$ \\
\hline Blood loss, ml, mean (SD) & $131(104)$ & $130(103)$ & $140(120)$ \\
\hline Use of hemostat agents & $53(24.8)$ & $47(24.2)$ & $6(30)$ \\
\hline Leak in intaraoperative flush test & $16(7.5)$ & $7(3.6)$ & $9(45)$ \\
\hline Difficult anastomosis & $10(4.7)$ & $7(3.6)$ & $3(15)$ \\
\hline Wide bladder neck & $14(6.5)$ & $11(5.7)$ & $3(15)$ \\
\hline Lymphadenectomy & $42(19.6)$ & $41(21.1)$ & $1(5)$ \\
\hline Hospitalization, days, mean (SD) & $1.3(0.7)$ & $1.3(0.8)$ & $1.3(0.6)$ \\
\hline Clavien 3 complications & $10(4.7)$ & $7(3.6)$ & $3(15)$ \\
\hline Bleeding complication & $11(5.1)$ & $7(3.6)$ & $4(20)$ \\
\hline Bloody urine & $13(6.1)$ & $5(2.6)$ & $8(40.0)$ \\
\hline Catherization, days & $9.1(5.2)$ & $8.0(2.3)$ & $19.8(10.5)$ \\
\hline Haematocrit drop & $0.05(0.07)$ & $0.05(0.08)$ & $0.07(0.03)$ \\
\hline Urinary tract infection, $\mathrm{N}(\%)$ & $4(1.9)$ & $4(2.1)$ & $0(0)$ \\
\hline pT3a or higher, N (\%) & $105(49.1)$ & $96(49.5)$ & $9(45)$ \\
\hline Lymph node metastasis, N (\%) & $9(4.2)$ & $8(4.1)$ & $1(5)$ \\
\hline Positive surgical marginal N (\%) & $63(29.4)$ & $58(29.9)$ & $5(25)$ \\
\hline \multicolumn{4}{|l|}{ Prostatectomy Gleason } \\
\hline$<7$ & $45(21)$ & $39(20)$ & $6(30)$ \\
\hline 7 & $146(68)$ & 134 (69) & $12(60)$ \\
\hline$>7$ & $23(11)$ & $21(11)$ & $2(10)$ \\
\hline
\end{tabular}

